\title{
Cluster Modelling for Cognitive Radio Ad-hoc Networks Using Graph Theory
}

\author{
N. Mansoor \\ Malaysia-Japan International Institute of Technology \\ (MJIIT) \\ Universiti Teknologi Malaysia (UTM) \\ Malaysia \\ A.K.M. M. Islam \\ Malaysia-Japan International Institute of Technology \\ (MJIIT) \\ Universiti Teknologi Malaysia (UTM) \\ Malaysia \\ M. Zareei \\ Malaysia-Japan International Institute of Technology \\ (MJIIT) \\ Universiti Teknologi Malaysia (UTM) \\ Malaysia
}

\author{
S. Baharun \\ Malaysia-Japan International Institute of Technology \\ (MJIIT) \\ Universiti Teknologi Malaysia (UTM) \\ Malaysia \\ S. Komaki \\ Malaysia-Japan International Institute of Technology \\ (MJIIT) \\ Universiti Teknologi Malaysia (UTM) \\ Malaysia
}

\begin{abstract}
With the swift expansion of wireless technologies, demand for radio spectrum is continuously mounting. Along with the spectrum scarcity problem, radio spectrums are also underutilized. Cognitive radio practices an open spectrum allocation technique, which can ensure efficient handling of the frequency bands. Suitable network model is a must for the implementation of cognitive radio networks. In this paper, an efficient cluster model for cognitive radio ad-hoc networks is presented using graph theory. The proposed clustering model is defined as a maximum edge biclique problem, where the spatial variations of spectrum availability are considered. This clustering scheme aims to maintain set of free common channels in every cluster, which allows smooth shifting among control channels. A parameter called Cluster Head Determination Factor (CHDF) is also introduced to select cluster-heads where clusters' operations are coordinated by cluster-heads. Each cluster comprises of a secondary clusterhead to combat the re-clustering issue for mobile nodes. Finally, simulation and comparative studies are conducted to evaluate the performance of the proposed method.
\end{abstract}

Keywords- cognitive radio networks; graph theory; ad-hoc networks; cluster-based network; re-clustering

\section{INTRODUCTION}

There is a rapid growth in wireless applications and technologies, which carries an ever-increasing demand for radio spectrums. However, radio spectrum is a limited natural resource and is almost fully distributed. Therefore, there lies spectrum scarcity problem for the forthcoming wireless technologies. On the other hand, due to the current command-and-control based spectrum allocation method, radio spectrum is underutilized with variance of frequency, time and space.

J. Mitola III introduces Cognitive Radio Network (CRN) aiming to use underutilized spectrum in an opportunistic manner [1, 2]. In real time, cognitive radio network, an intelligent wireless communication system, has the ability to adjust itself on the situation and to make relevant changes in operating parameters such as carrier frequency, transmitpower, modulation strategy, etc. In CRN, licensed users are considered as Primary Users (PUs) and Secondary Users (SUs) are the unlicensed users who use the free spectrum opportunistically.

A decentralized and self-configured wireless network is considered to be a wireless ad-hoc network, in which the network does not depend on established infrastructures [3, 4]. Mobile Ad-hoc Networks (MANETs) and Wireless Sensor Networks (WSN) are the two popular types of wireless adhoc networks [4-6]. However, both of these two technologies use fixed spectrums, where users are prohibited to dynamically use the unused spectrum. Due to the flexible and dynamic spectrum usage behaviour over other ad-hoc technologies, CRN has received a profound interest to communication network researchers for the past few years.

In this paper, efficient cluster-based model for cognitive radio ad-hoc network is presented using graph theory, where 
the spatial variations of spectrum availability are considered for clustering. Set of free common channels inhabits every cluster to allow smooth shifting among control channels. A parameter called Cluster Head Determination Factor (CHDF) is introduced to select cluster-head where intra-cluster operation is coordinated by cluster-head. Each cluster having a secondary cluster-head helps to combat the re-clustering issue for mobile nodes. The components of the proposed cluster-based model are Cluster-Heads (CHs), Secondary Cluster-Heads (SCHs), Cluster Members (CMs), and Forwarding Nodes (FNs). Simulation results show that the proposed cluster-based model outperforms other recently developed clustering approaches by upholding a reduced number of clusters in the network where number of common channels in each cluster also remains steady.

This paper is organized as follows. In section 2, a brief analysis on different recently developed networks for ad-hoc CRN is presented. The proposed cluster-based model for cognitive radio ad-hoc network is described in section 3 . Simulation results and comparative study are conducted and is presented in section 4. Conclusion and future works have been discussed in section 5 .

\section{RELATED WORKS}

Cognitive radio network has been receiving profound interest to communication network researchers for the past few years. This section discusses various recent cluster-based networks for ad-hoc CRN.

Spectrum awareness and local control channel assignment are the two main concerns for the reviewed networks. A cluster formation algorithm based on available channels, physical location and spectrum occupancy history is proposed in [7] that suffers from re-clustering issue for varying spectrum availability and for mobility of nodes. A distributed cluster agreement algorithm called SpectrumOpportunity Clustering (SOC) is proposed in [8] where clusters are formed based on common idle channels. Although the method provides desirable balance between common channels and cluster size, however, [8] can produce a huge number of clusters. Moreover, re-clustering for mobile nodes is another shortfall for SOC [8].

A degree based clustering method is presented in [9] where 2-hop communication is considered within a cluster. Every node in the network requires extra processing power as node maintains complete network information. Moreover, once the node is mobile, the re-clustering issue becomes acute in [9]. Proposed cluster-based network in [10] forms clusters which considers changing spectrum availability. However, the scheme adds extra delay in intra-cluster communication as cluster size can be huge and re-clustering is essential for mobility of nodes in [10]. ID based clustering protocol for CRN is presented in [11], where a buffering technique is used to reduce the packet loss. Re-clustering issue is dominant in this scheme both for varying spectrum availability and for mobile nodes.

A widely conversed cluster-based CRN named CogMesh presented in [12] constructs clusters around a specific local channel called master channel. The scheme in [12] has some provision for nodes' mobility. However, not all the clusters have the mechanism to deal with the re-clustering issue for mobile nodes. A Dynamic clustering scheme for cognitive radio ad-hoc network is presented in [13]. Re-clustering issue is dominant in this scheme both for varying spectrum availability and for mobile nodes. Nodes' mobility is considered in cluster-based scheme for CRN [14]. However, the network suffers from frequent re-clustering problem with varying spectrum availability.

These existing CRN models have several drawbacks, which hinder the rapid development of CRN. Thus, a stable network in terms of varying spectrum and nodes' mobility is deemed necessary for CRN. This leads to the development of a model, which is discussed in the following section.

\section{THE MODEL}

\section{A. Network Environment}

The assumed ad-hoc networks comprised of selforganized Cognitive Radios (CRs)/ Secondary Users (SUs), where SUs have the capability to sense and utilize available free spectrums independently. In the network, SUs are location aware and coexist with PUs. The CRs have the processing capability to calculate own CHDF value. CRs are also aware of the CHDF values of their neighboring CRs.

The spectrum band is divided into non-overlapping orthogonal channels with unique channel ID for each channel. Licensed spectrum of the PU is only accessible to SUs if the PU's transmission is absent. Subject to the physical location, channel availability varies for SUs. We consider that a SU detects available spectrum by sensing free frequency bands using methods such as energy detectors, cyclostationary feature extraction, or eigenvalue-based feature extraction [15]. The proposed clustering mechanism is autonomous to any specific PU activity model. Semi-Markov ON- OFF model to evaluate the performance of the proposed architecture is considered. Semi-Markov ON-OFF process is modeled on any channel for the PU traffic. Busy (ON) or idle (OFF) are the two states that have been considered for any channel [16]. The activation period of any channel is assumed to be an independent random variable. This assumption is realistic when spectrum bands are licensed to independently operating PUs (e.g., channels operated by different TV stations).

IEEE 802.22 standard for the operating frequencies of the system is considered, where SU uses a free channel opportunistically and vacates the channels whenever PUs presence is sensed. To avoid interference with PUs, it is assumed that a simple interference avoidance model is present in the system. Here, there are two transceivers in each CR are considered, where one is used for control and the other one is used for data transmission. With the ability for least switching delay, each transceiver is spectrum aware. Equal transmission range is considered for all the cognitive radios. A link exists between two radios iff they are in each other's communication range and share at least one common channel. It was also assumed that there is a global common control channel exists in the network. Each cluster declares 
its own control channel once the cluster formulation is completed. Intra-cluster and inter-cluster communications are coordinated by the Cluster Heads (CHs). For inter-cluster communication, Forwarding Nodes (FNs) are used. FNs are those nodes that are positioned at the edge of two neighboring clusters and can hear beacons from both clusters.

\section{B. Cluster Formation}

Once node completes spectrum sensing, it prepares the accessible channel list (ACL), which is the resultant channel generated from this process. For an example, a particular node $v$ senses free channels listed as channel 1, 2, 4, 6 and prepares its ACL, $C_{v}=\{1,2,4,6\}$. Thus, each of the nodes in a network exhibits its own ACL. Next, nodes share ACL, $C_{i}$ and neighbors list, $N_{i}$ among 1-hop neighbors (where $i=$ $1,2,3, \ldots, n$ ). Figure 1 (a) shows the connectivity graph of a cognitive radio ad-hoc network with the accessible channel list associated with each node is denoted in the brackets.

Initially, based on neighbor list $N_{i}$ and ACL $C_{i}$, every $C R_{L}$ constructs an undirected bipartite graph

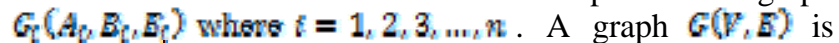
called bipartite if vertices set $V$ can be split into two disjoint sets $A$ and $B$, where $A U B=F$, such that all edges in $E$ connect vertices from $A$ to $B$. For a node $C R_{i}, A_{l}=C R_{l} U N_{l}$ and $B_{l}=C_{i}$ where, $C_{i}$ is the ACL of $C R_{i}$. An edge $(x, y)$ exists between vertices $x \in A_{l}$ and $y \in B_{l}$ if $y \in C_{l}$, i.e., channel $y$ is in the ACL of $C R_{?}$. A bipartite graph $G_{Q}\left(A_{Q}, B_{Q}, E_{\Omega}\right)$ constructed by $C R_{\Omega}$ from Figure 1(a) is shown in Figure 1(b). The set of vertices $A_{a}$ corresponds to the 1-hop neighbors $N_{a}=\{b, c, d, \in, f\}$ plus $a$, while the set of vertices $B_{a}$ corresponds to the accessible channels list of $C R_{\alpha}$, which is $C_{\alpha}=\{1,2,3,4,6,7,8,9\}$. Here, vertex $a$ of $A_{a}$ is connected to all vertices in $B_{a}$, since $B_{\Omega}=C_{\Omega}$.

The proposed clustering scheme is defined as a maximum edge biclique problem. The proposed clustering scheme attempts to include maximum nodes in each cluster while ensuring enough number of common channels for intracluster communication. The maximum edge biclique graph of a node can be constructed from the bipartite graph of that particular node. In Figure 1(c), the maximum edge biclique graph of node $a$ is presented, which is constructed from bipartite graph of node $a$ in Figure 1(b). Node $a$ forms its maximum edge biclique graph with neighboring nodes $b, c$ and $f$ and channels $(1,3,6,9)$. Thus, every individual node in the network constructs own maximum edge biclique graph.

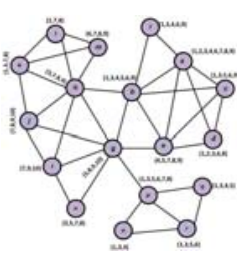

(A)

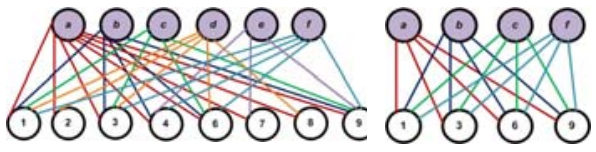

(B)

(C)

\section{FIGURE I. (A) CONNECTIVITY GRAPH OF A COGNITIVE RADIO NETWORK WITH THE ACLS IN THE BRACKETS, (B) BIPARTITE GRAPH CONSTRUCTED BY NODE A, (C) MAXIMUM EDGE BICLIQUE GRAPH OF NODE A.}

New $C_{i}$ and $N_{i}$ values will then be determined by the maximum edge biclique graph. To determine a cluster, cluster head selection is a vital task. Therefore, a parameter called Cluster Head Determination Factor (CHDF) is introduced to select these cluster heads. Since, new $C_{i}$ and $N_{i}$ values play a very important role in the system, thus they could be the determining factor for the selection of the cluster heads. Hence, these two parameters are chosen to determine the CHDF value. Several relations between these two parameters have been examined; however more importance is given on $C_{i}$ over $N_{i}$. This provides more robustness for intra-cluster communication, as higher number of reserved channels provides more flexibility for channel switching and reduces re-clustering issue for changing spectrum availability. The root operator is used to downsize the magnitude of the CHDF value as shown in equation (1).

$$
C H D F_{i}=\sqrt[C_{i}]{C_{i}^{N_{i}}} ; i=1,2,3 \ldots n
$$

Every CR calculates CHDF based on equation (1) and is presented in Figure 2(a). A node declares itself as cluster head if its own CHDF value is higher than all its neighbors. Once the CHDF value of a node $C R_{i}$ is lesser than any of its neighbor, $C R_{i}$ joins the neighboring node that has the highest value as cluster member (CM). After the cluster formation, $\mathrm{CH}$ selects $\mathrm{SCH}$ from the CMs based on the CHDF value. The SCH takes charge of the cluster if current $\mathrm{CH}$ moves out, which shrinks the possibility of re-clustering. The proposed cluster-based network is presented in Figure 2 (b). 


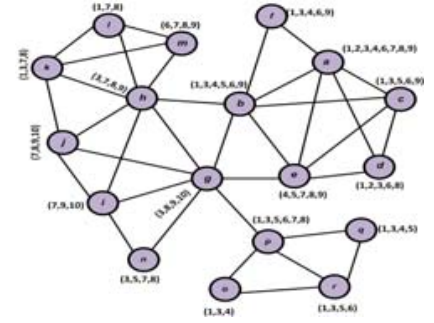

(A)

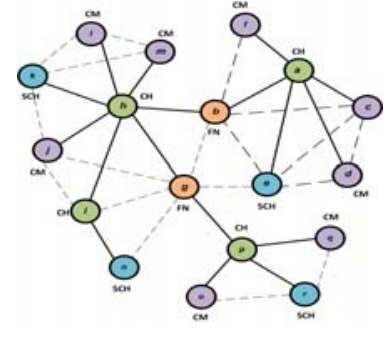

(B)
FIGURE II. (a) CHDF VALUE FOR EACH NODE, (b) PROPOSED CLUSTER-BASED NETWORK.

In Figure 2(b) solid line denotes the logical link and dotted line denotes physical links. $\mathrm{CH}$ defines and upholds operating channels for the cluster. To find the existence of any other clusters in the neighborhood, CMs check their neighbor list for other cluster heads. CM becomes the FN and connects two clusters once it finds other $\mathrm{CH}$ in the neighbor list. In this proposed cluster-based scheme, cluster consists of one $\mathrm{CH}$, one SCH and CMs. All cluster members are 1-hop apart from the $\mathrm{CH}$. FN connects two neighboring clusters, where there can be maximum two intermediate FNs between two CHs. Using local common channels, intracluster communications are performed.

To evaluate the performance of the model, simulation is conducted. Simulation results are then compared to the other similar networks that have been established. This is discussed in the following section.

\section{SiMULATION RESUlTS}

MATLAB is used as a simulation tool to evaluate the performance of the proposed cluster-based model. This simulation result in terms of clusters is then compared to the other three existing approaches which have similar considerations as this proposed network, namely, clusterbased approach in [7], spectrum opportunity-based control channel (SOC) approach in [8], and CogMesh [12]. The simulation area is $10 \mathrm{~km}^{2}$ (square kilo-meters), where the cognitive nodes are positioned randomly in the simulation environment. The communication range for each node to 500 meters and 10 available channels in the simulation environment is considered. This is shown in Figure 3.

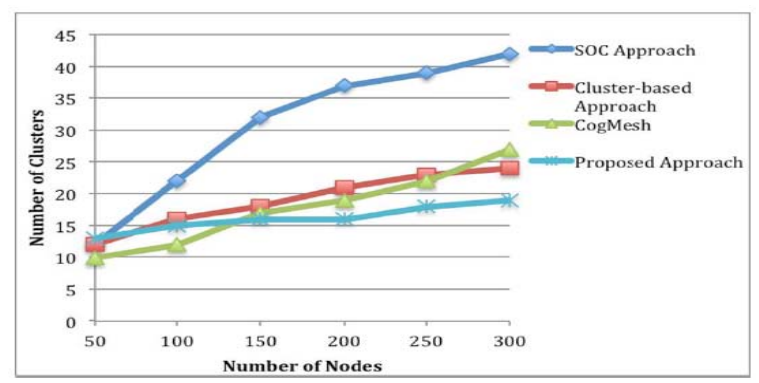

FIGURE III. PERFORMANCE COMPARISON OF THE PROPOSED ARCHITECTURE WITH OTHER APPROACHES (IN TERMS OF NUMBER OF CLUSTERS).
Figure 3 shows that as the number of nodes increases, the number of clusters also increases for all approaches. Here, in a network of 50 nodes, the proposed model constructs 13 clusters, while cluster-based approach, SOC approach, and CogMesh construct 12 clusters, 12 clusters and 10 clusters respectively. When the node's number upturns to 300, proposed architecture gives 19 clusters, while cluster-based approach, SOC approach and CogMesh create 24 clusters, 42 clusters and 27 clusters respectively. With the increasing number of nodes, the SOC approach generates higher number of clusters as compared to the other three approaches. It can be seen that the proposed model constructs lesser number of clusters with the increasing number of nodes. Thus, this model provides a better description for cognitive radio ad-hoc network in terms of communication as compared to the others.

\section{CONCLUSION AND FUTURE WORKS}

In this paper, a spectrum aware cluster-based network has been successfully developed that breaks the CRN ad-hoc network into clusters. This paper also introduces a parameter named CHDF, which is used to select cluster heads. This is materialized through the concept of bipartite and biclique in graph theory. Through the comparison between the performances of this model and other existing models has shown that this proposed model performs better. Moreover, the proposed clustering scheme attempts to include maximum nodes in each cluster while ensuring enough number of common channels for intra-cluster communication. The next research step is to develop routing and broadcasting protocols for the proposed network.

\section{ACKNOWLEDGMENT}

This research is partially supported by University Research Grant of Universiti Teknologi Malaysia GUP Tier 1 with Vote No. 05H61 and GUP Tier 2E with Vote No.09J73 of Ministry of Higher Education (MoHE) year 2014 to 2015.

\section{REFERENCES}

[1] I. F. Akyildiz, W. Y. Lee, M. C. Vuran, and S. Mohanty, "NeXt generation/dynamic spectrum access/cognitive radio wireless networks: A survey," Computer Networks, vol. 50, pp. 2127-2159, Sep 152006.

[2] J. Mitola and G. Q. Maguire, "Cognitive radio: Making software radios more personal," IEEE Personal Communications, vol. 6, pp. 13-18, August 1999

[3] C. K. Toh, Ad hoc mobile wireless networks: protocols and systems: Pearson Education, 2001.

[4] A. K. M. Muzahidul Islam, J. Uchida, W. Chen, and K. Wada, "A Better Dynamic Cluster-based Structure Wireless Sensor Network for Efficient Routing," International Journal of Innovative Computing, Information and Control (IJICIC), vol. 8, 2012.

[5] J. Uchida, A. K. M. Muzahidul Islam, Y. Katayama, W. Chen, and K. Wada, "Construction and Maintenance of a Novel Cluster-based Architecture for Ad Hoc Sensor Networks," Adhoc \& Sensor Wireless Networks, vol. 6, 2008.

[6] A. K. M. Muzahidul Islam, K. Wada, and W. Chen, "Dynamic Cluster-based Architecture and Data Congregation Protocols for 
Wireless Sensor Network," International Journal of Innovative Computing, Information and Control (IJICIC), vol. 9, 2013.

[7] X. Li, F. Hu, H. Zhang, and X. Zhang, "A Cluster-Based MAC Protocol for Cognitive Radio Ad Hoc Networks," Wireless Personal Communications, vol. 69, pp. 937-955, 2013/03/01 2013.

[8] L. Lazos, S. Liu, and M. Krunz, "Spectrum opportunity-based control channel assignment in cognitive radio networks," presented at the Proceedings of the 6th Annual IEEE communications society conference on Sensor, Mesh and Ad Hoc Communications and Networks, Rome, Italy, 2009.

[9] J.-z. Zhang, F. Wang, F.-q. Yao, H.-s. Zhao, and Y.-s. Li, "Clusterbased distributed topology management in Cognitive Radio Ad Hoc networks," in Computer Application and System Modeling (ICCASM), 2010 International Conference on, 2010, pp. V10-544V10-548.

[10] A. Asterjadhi, N. Baldo, and M. Zorzi, "A cluster formation protocol for cognitive radio ad hoc networks," in Wireless Conference (EW), 2010 European, 2010, pp. 955-961.

[11] C.-C. Tang, K.-F. Ssu, and C.-H. Yang, "A Cluster-Based Link Recovery Mechanism for Spectrum Aware On-Demand Routing in Cognitive Radio Ad Hoc Networks," in Advances in Intelligent Systems and Applications-Volume 1, ed: Springer, 2013, pp. 601-610.

[12] T. Chen, H. G. Zhang, G. M. Maggio, and I. Chlamtac, "CogMesh: A cluster-based cognitive radio network," 2007 2nd Ieee International Symposium on New Frontiers in Dynamic Spectrum Access Networks, Vols 1 and 2, pp. 168-178, 2007.

[13] N. Mansoor, A. K. M. M. Islam, S. Baharun, S. Komaki, and K. Wada, "CoAd: A cluster based adhoc cognitive radio networks architecture with broadcasting protocol," in Informatics, Electronics \& Vision (ICIEV), 2013 International Conference on, 2013, pp. 1-6.

[14] A. C. Talay and D. T. Altilar, "United nodes: cluster-based routing protocol for mobile cognitive radio networks," Iet Communications, vol. 5, pp. 2097-2105, Oct 142011.

[15] T. Yucek and H. Arslan, "A survey of spectrum sensing algorithms for cognitive radio applications," Communications Surveys \& Tutorials, IEEE, vol. 11, pp. 116-130, 2009.

[16] A. Motamedi and A. Bahai, "MAC Protocol Design for Spectrumagile Wireless Networks: Stochastic Control Approach," in New Frontiers in Dynamic Spectrum Access Networks, 2007. DySPAN 2007. 2nd IEEE International Symposium on, 2007, pp. 448-451. 